\title{
Escalla de temor a la COVID- 19: validación de la versión en español en la población mexicana
}

\author{
Alejandro I. Soto-Briseño1, Rita A. Gómez-Díaz²*, Adriana L. Valdez-González², \\ Ricardo C. Saldaña-Espinoza², José J. Favila Bojórquez y Niels H. Wacher \\ ${ }^{1}$ Servicio de Psiquiatría; ' Unidad de Investigación en Epidemiología Clínica. Unidad Médica de Alta Especialidad, Hospital de Especialidades, \\ Centro Médico Siglo XXI, Instituto Mexicano del Seguro Social, Ciudad de México, México
}

\section{Resumen}

Introducción: La Escala de temor a la COVID-19 (FCV-19S) se usa en el tamizaje de síntomas de ansiedad y depresión relacionados con la COVID-19 en población general; consta de siete preguntas con respuestas tipo Likert (1-5). Nuestro objetivo fue validar la versión del FCV-19S en la población general mexicana. Material y métodos: Diseño transversal analítico. Se incluyeron 306 sujetos de la población general durante 2020 con firma previa de consentimiento informado. Se aplicaron pruebas de esfericidad de Barlett y Kaiser-Meyer-Olkin (KMO). Se calculó la confiabilidad con el alfa de Cronbach, la validez externa utilizando la Escala hospitalaria de ansiedad y depresión y el coeficiente de correlación de Pearson para retest. Resultados: La muestra de la población general incluyó a 306 participantes, el 64.4\% mujeres ( $n=197)$, edad media 32 años (18-68). Obtuvimos un KMO $=0.848$, consistencia interna con alfa de Cronbach $=0.870$ (IC 95\%: 0.848-0.891), coeficiente rho de 0.508 ( $p$ < 0.001) y validez externa de $0.151(p=0.008)$. El análisis confirmatorio mostró: $\chi^{2}=22.802$ (df $\left.=13\right)$ con $C M I N-D F=1.900, p \leq 0.001, \mathrm{GFI}=0.972, \mathrm{CFI}=0.901, \mathrm{RMSEA}=0.062$ (IC 90\%: 0.019-0.100) y TLI=0.827. Conclusiones: $\mathrm{La}$ FCV-19S demuestra propiedades psicométricas adecuadas (confiabilidad, consistencia interna, correlación con mediciones subsecuentes y validez de convergencia) para su aplicación en la población general mexicana.

PALABRAS CLAVE: COVID-19. Escala de temor a COVID-19. Español. Psicométricos. México.

\section{Fear of COVID-19 scale: validation in Spanish in the Mexican general population}

\section{Abstract}

Introduction: The fear of COVID-19 scale (FCV-19S) is used to screen for symptoms of anxiety and depression related to COVID-19 in the general population; it consists of seven questions with Likert-type answers (1-5). Our objective was to validate FCV-19S Spanish version in the Mexican general population. Material and methods: Analytical, cross-sectional design. Three-hundred and six subjects from the general population were included during 2020 after having signed informed consent. Barlett and Kaiser-Meyer-Olkin (KMO) sphericity tests were applied. Reliability was calculated with Cronbach's alpha, and external validity, using the Hospital Anxiety and Depression Scale and Pearson's correlation coefficient for retest. Results: The general population sample included 306 participants; $64.4 \%$ were women $(n=197)$, mean age was 32 years (18-68). We obtained a KMO =0.848, internal consistency with Cronbach's alpha $=0.870$ (95\% Cl: 0.848-0.891), a rho coefficient of 0.508 $(p<0.001)$ and external validity of $0.151(p=0.008)$. Confirmatory analysis showed: $\square 2=22.802(d f=13)$ with CMIN-DF = 1.900 ( $p \leq 0.001), \mathrm{GFI}=0.972, \mathrm{CFI}=0.901, \mathrm{RMSEA}=0.062(90 \% \mathrm{Cl}: 0.019-0.100)$ and $\mathrm{TLI}=0.827$. Conclusions: According to our findings, the scale shows adequate psychometric properties: reliability, internal consistency, correlation of subsequent measurements and convergence validity, for initial screening of Mexican general population.

KEY WORDS: COVID-19. Fear of COVID-19 scale. Spanish. Psychometrics. Mexico.

Correspondencia:

*Rita A. Gómez-Díaz

E-mail: ritagomezdiaz@yahoo.com.mx
Gac Med Mex. 2021;157:586-593

Disponible en PubMed

www.gacetamedicademexico.com

0016-3813/@ 2021 Academia Nacional de Medicina de México, A.C. Publicado por Permanyer. Este es un artículo open access bajo la licencia CC BY-NC-ND (http://creativecommons.org/licenses/by-nc-nd/4.0/). 


\section{Introducción}

Considerando los casos de neumonía asociados al coronavirus 2 del síndrome respiratorio agudo grave (SARS-CoV-2) y su enfermedad, la pandemia por COVID-19 constituye un problema de salud pública con tasas variables de morbimortalidad ${ }^{1-3}$.

De acuerdo con la Dirección General de Epidemiología, en nuestro país contamos con 2,175, 662 casos confirmados acumulados de COVID19 (49.9\% mujeres) hasta la primera quincena de marzo 2021; lo cual corresponde al $17.26 \%$ de los 126,014,024 habitantes de nuestro país según el último reporte del Instituto Nacional de Estadística y Geografía (INEGI)4. Se reportan 32,206 casos activos, de los cuales el $18.71 \%$ requieren tratamiento intrahospitalario; las principales comorbilidades asociadas son hipertensión arterial (17.31\%), obesidad $(14.45 \%)$, diabetes mellitus (13.35\%) y tabaquismo (7.35\%); además, se reportan 195,908 muertes acumuladas $^{5-7}$. Durante los meses de enero a agosto del 2020, en nuestro país la COVID-19 fue la segunda causa de muerte ${ }^{8}$.

Existe miedo y preocupación al contacto con otras personas por el riesgo de infección por SARS-CoV-2, desarrollar formas severas de COVID-19, la posibilidad de requerir ventilación mecánica, perder seres queridos o morir. Las medidas de distanciamiento social son indispensables para reducir las tasas de infección, sin embargo, se asocian a síntomas de ansiedad y depresión que afectan especialmente a grupos vulnerables como mujeres embarazadas, niños, personas con discapacidad física o mental y adultos mayores ${ }^{9,10}$

La escala de temor a la COVID-19 (FCV-19S) es un instrumento utilizado para la detección del temor relacionado con la COVID-19 en la población general, que identifica síntomas de ansiedad y depresión relacionados con esta pandemia. Consta de siete elementos, con respuestas tipo Likert (1-5) y rangos de puntuación de 7 a 35. Cuanto mayor sea la puntuación, mayor será el miedo relacionado con la COVID19. Este instrumento ha mostrado consistencia interna en su versión original, con $\alpha=0,82$, y fiabilidad en la repetición del instrumento (coeficiente intraclase de correlación $=0.72)^{11}$. Hasta la fecha se han validado versiones y reportado su consistencia interna en varios idiomas, como italiano $(\alpha=0.87)^{12}$, ruso $(\alpha=0.81)^{13}$, turco $(\alpha=0.85)^{14}$, árabe $(\alpha=0.88)^{15}$, hebreo $(\alpha=0.86)^{16}$, bengalí $(\alpha=0.87)^{17}$, malayo $(\alpha=0.89)^{18}$, rumano $(\alpha=0.88)^{19}$, portugués $(\alpha=0.88)^{20}$, japonés $(\alpha=0.87)^{21}$, griego $(\alpha=0.83)^{22}$ e inglés $\left(\alpha=0.84\right.$ y 0.86 en diferentes muestras) ${ }^{23}$.

En español ha sido validado en la población general en Perú ( $n=832$; edad media: 38,37 $\pm 12,75$ años), reportando una $\alpha=0.87^{24}$, y en estudiantes universitarios de 18 a 40 años en España ( $n=606$; edad media: $21.59 \pm 3.04$ años), con un $\alpha=0.86^{25}$.

En nuestro país, la FCV-19S ha sido utilizada para realizar mediciones del temor relacionadas con la COVID-19 en trabajadores de la salud (con $\alpha=0.92$ ); sin embargo, no se realizaron mediciones de convergencia ${ }^{26}$.

Teniendo en cuenta lo anterior, nuestro objetivo fue validar la versión en español de la FCV-19S en la población general mexicana.

\section{Material y métodos}

En este estudio, la versión original del FCV-19S fue traducida al español por un traductor bilingüe certificado independiente con amplia experiencia en las áreas clínicas, posteriormente revisada por dos traductores bilingües independientes para confirmar sus características (inteligibilidad y fidelidad) y se realizó la retrotraducción.

La población del estudio fue la población general de la Ciudad de México, se utilizó una estrategia de muestreo por conveniencia. El reclutamiento se realizó por medio de redes sociales, se incluyeron 306 participantes de 18 a 80 años, previa firma y envío por correo electrónico del consentimiento informado de participación. La información fue recopilada durante los meses de agosto a noviembre de 2020, por medio de llamadas telefónicas cuando se establecieron las medidas de distanciamiento social a nivel nacional para reducir los riesgos de infección entre los participantes y el personal médico.

Se recopiló información sociodemográfica, incluyendo edad, sexo, estado civil, nivel académico y estado socioeconómico.

Para determinar la validez de la convergencia de la FCV-19S en la versión original, se utilizó la Escala de ansiedad hospitalaria y depresión (HADS), la cual ha sido validada previamente en nuestro país ${ }^{25,26}$.

\section{Análisis estadístico}

Estadística descriptiva de los elementos de la FCV$19 S$ (media y desviación estándar de los elementos principales) y la matriz policórica de sus siete 
elementos; el análisis factorial se realizó mediante la prueba de esfericidad de Bartlett y la prueba KeiserMeyer-Olkin (KMO). El criterio de validez de la FCV$19 S$ en su versión en español se determinó con el coeficiente de correlación de Pearson utilizando la HADS. La fiabilidad de la escala fue examinada mediante fiabilidad compuesta (CR), la consistencia interna de la puntuación global se calculó utilizando el coeficiente alfa de Cronbach. La bondad del ajuste del modelo se examinó con los siguientes índices: el índice de bondad de ajuste (GFI), la prueba chi-cuadrada $\left(\chi^{2}\right)$, los grados de libertad (df), el error de aproximación cuadrático medio (RMSEA), el índice de ajuste comparativo (CFI), el residuo estandarizado cuadrático medio (SRMR) y el índice Tucker-Lewis (TLI). El análisis se llevó a cabo utilizando SPSS v.23.0 (IBM Corp., Armonk, NY), FACTOR v.10.10.03 (Ferrando y Lorenzo-Seva, Universitat Rovira i Virgili) e IBM SPSS AMOS v.23 (IBM Corp., Armonk, NY).

\section{Resultados}

\section{Características sociodemográficas}

La muestra de la población general incluyó a 306 participantes ( $n=197,64.4 \%$ mujeres), con una edad media de 32 años (18-68); al agrupar por edad, el mayor porcentaje (44.4\%) se encontró entre los 30 y 39 años. En cuanto al nivel académico, agrupamos los resultados en cinco grupos y respecto al estado civil encontramos: $21.8 \%$ solteros, $49.4 \%$ casados, $9.8 \%$ divorciados y $18.9 \%$ en unión libre (Tabla 1).

\section{Análisis psicométrico de la versión en español de la FCV-19S}

La puntuación media del total del puntaje de la FCV19S fue de 17.63 (desviación estándar $[D E] \pm 6.60$ ) (Tabla 2). Se realizó una prueba t para muestras independientes para comparar las puntuaciones FCV-19S entre las mujeres 19.09 (DE \pm 6.67 ) y los hombres 14.99 (DE \pm 5.62 ), con una diferencia significativa $(\mathrm{t}=-4.01 ; \mathrm{p}<0.001)$.

Las siete preguntas de la FCV-19S (Fig. 1) se distribuyeron asimétricamente con las frecuencias más altas en los rangos inferiores, excepto las preguntas 1 y 7 , con una distribución forma no normal (prueba de Kolmogorov-Smirnov, $p<0.001$ ).

El análisis factorial exploratorio se realizó en 70 participantes escogidos al azar, resultando en un $\mathrm{KMO}=0.848$ (intervalo de confianza del 95\%
Tabla 1. Características de la muestra $(\mathbf{n}=306)$

$\begin{array}{lll}\text { Sexo } n=306(\%) & \text { Nivel educativo } & \text { Estado civil } \\ \text { Mujeres 197 (64.4) } & \text { Primaria 6 (2.0) } & \text { Soltero 67 (21.8) } \\ \text { Hombres } & \text { Secundaria 51 (16.7) } & \text { Casado 151 (49.4) } \\ 109(35.6) & \text { Bachillerato 78 (25.5) } & \text { Divorciado 30 (9.8) } \\ \text { Edad (años) (\%) } & \text { Universidad 126(41.1) } & \text { Unión libre 58 (18.9) } \\ 18-29126(41.1) & \text { Posgrado 12 (3.9) } & \\ 30-39136(44.4) & \text { Ninguna 8 (2.6) } & \\ 40-4931(10.1) & \text { No contestaron } & \\ 50-5911(3.5) & \text { 25(8.2) } & \\ \geq 602(0.7) & & \end{array}$

[IC 95\%]: 0.848-0.891), la prueba de esfericidad del Barlett fue significativa $\left(\chi^{2}: 272.0 ; p<0.001\right)$, lo cual indica que el análisis factorial es pertinente para el conjunto de datos. Todos los elementos fueron estadísticamente significativos para la correlación policórica (mín.: 0.258, máx.: 0.727, $p<0.001$ ) (Tabla 3).

El análisis de componentes principales extrajo un componente con valor propio (4.2744) que explicaba el $61 \%$ de la varianza. La carga factorial de todas las preguntas osciló entre 0.519 y 0.869 , comunalidad superior a 0.3 (Tabla 4).

Posteriormente, se realizó el análisis factorial confirmatorio con 236 participantes, diferentes de los utilizados para el análisis factorial exploratorio, utilizando el programa AMOS. Se analizaron varios modelos con el fin de identificar el que mejor se ajustaba a los índices de validación recomendados. El modelo 1 del análisis factorial confirmatorio presentó las siete preguntas y no se correlacionaron las desviaciones de error. El valor de RMSEA fue 0.090, dentro de los valores establecidos como satisfactorios (Fig. 2). En nuestra validación en español encontramos una interrelación entre las preguntas 3 (mis manos sudan cuando pienso en coronavirus-19) y 6 (no puedo dormir porque estoy preocupado(a) por enfermarme de coronavirus-19), con las puntuaciones más bajas, por lo que el modelo 2 se realizó teniendo en cuenta la variación del error (Fig. 3); además, encontramos una interrelación entre las preguntas 6 (no puedo dormir porque estoy preocupado (a) por enfermarme de coronavirus-19) y 7 (mi corazón se acelera cuando pienso en enfermarme con coronavirus-19), con una puntuación moderada pero similar entre las preguntas 6 y 7 ; por lo tanto, se creó el modelo 3 , considerando una variación del error (Fig. 4). Los resultados del modelo 3 se ajustaron satisfactoriamente a los índices recomendados con los siguientes resultados: $\chi^{2}=22.802(\mathrm{df}=13)$ 
Tabla 2. Análisis descriptivo de las siete preguntas de la versión en español de la Escala de Temor a la COVID-19

\begin{tabular}{|l|l|l|l|l|l|}
\hline & Media & Intervalo de confianza 95\% para la media & Varianza & Sesgo & Curtosis \\
\hline Ítem 1 & 2.96 & $2.77-3.14$ & 1.363 & -0.328 & -0.937 \\
\hline Ítem 2 & 2.44 & $2.25-2.63$ & 1.466 & 0.308 & -1.062 \\
\hline Ítem 3 & 1.72 & $1.58-1.86$ & 0.741 & 0.943 & -0.048 \\
\hline Ítem 4 & 2.57 & $2.36-2.79$ & 1.874 & 0.201 & -1.352 \\
\hline Ítem 5 & 2.57 & $2.37-2.78$ & 1.708 & 0.222 & -1.202 \\
\hline Ítem 6 & 1.77 & $1.62-1.92$ & 0.947 & 1.237 & 1.085 \\
\hline Ítem 7 & 2.19 & $2.01-2.37$ & 1.335 & 0.528 & -0.815
\end{tabular}

Tabla 3. Matriz estandarizada de la varianza/covarianza (correlación policórica)

\begin{tabular}{|c|c|c|c|c|c|c|c|}
\hline Correlaciones & Pregunta 1 & Pregunta 2 & Pregunta 3 & Pregunta 4 & Pregunta 5 & Pregunta 6 & Pregunta 7 \\
\hline Pregunta 1 & 1.000 & & & & & & \\
\hline Pregunta 2 & $0.500^{*}$ & 1.000 & & & & & \\
\hline Pregunta 3 & $0.425^{\star}$ & $0.711^{*}$ & 1.000 & & & & \\
\hline Pregunta 4 & $0.552^{*}$ & $0.450^{*}$ & $0.367^{*}$ & 1.000 & & & \\
\hline Pregunta 5 & $0.549^{*}$ & $0.700^{*}$ & $0.526^{*}$ & $0.480^{*}$ & 1.000 & & \\
\hline Pregunta 6 & $0.449^{*}$ & $0.727^{\star}$ & $0.709^{*}$ & $0.258^{*}$ & $0.512^{*}$ & 1.000 & \\
\hline Pregunta 7 & $0.489^{*}$ & $0.618^{*}$ & $0.661^{*}$ & $0.335^{*}$ & $0.628^{*}$ & $0.668^{*}$ & 1.000 \\
\hline
\end{tabular}

${ }^{*} \mathrm{p}<0.001$.
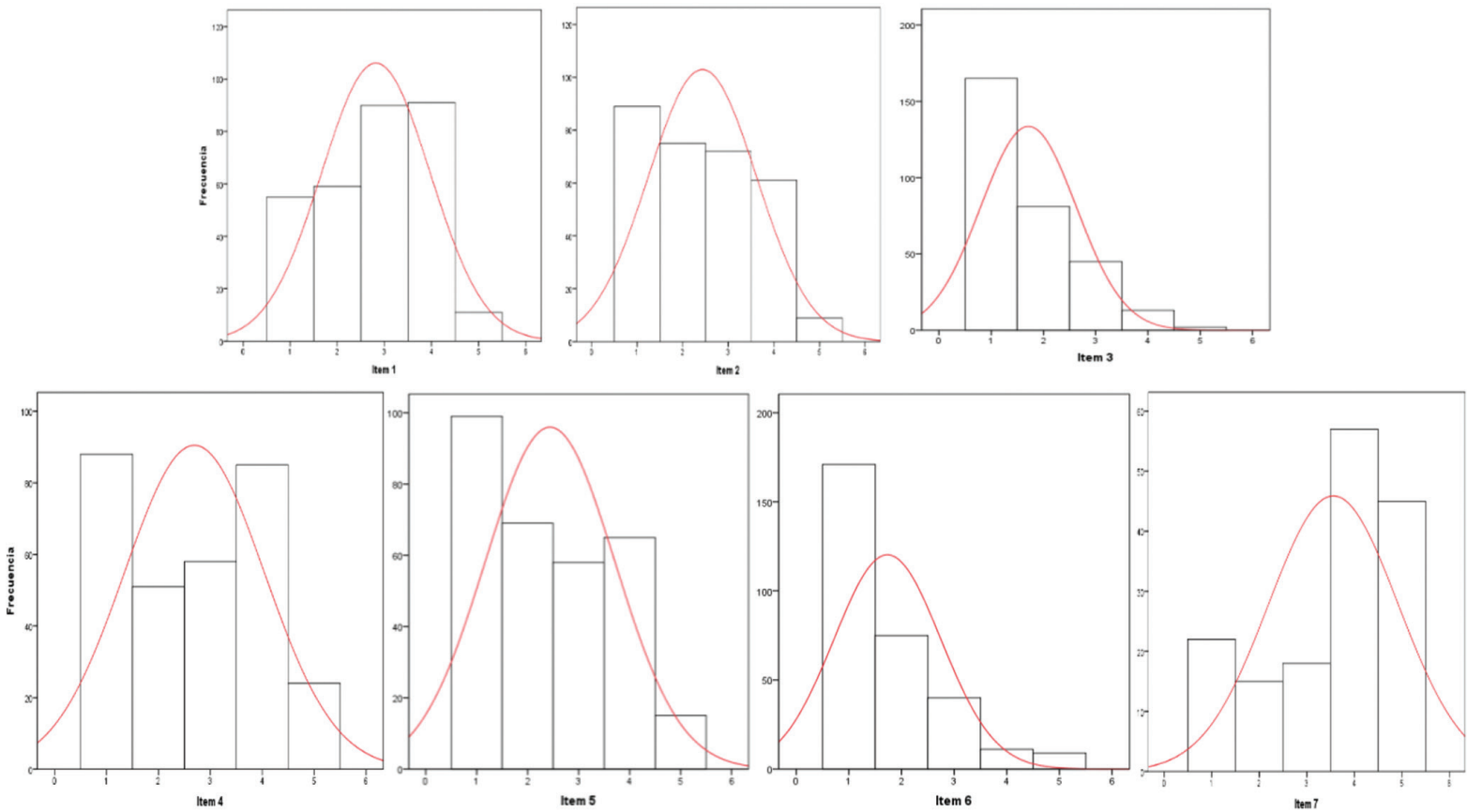

Figura 1. Histogramas de las siete preguntas de la versión en español de la Escala de temor a la COVID-19 (las líneas rojas muestran la distribución normal de la curva). 
Tabla 4. Matriz factorial sin rotamiento

\begin{tabular}{|l|c|c|}
\hline Variable & F1 & Comunalidad \\
\hline Pregunta 1 & 0.640 & 0.410 \\
\hline Pregunta 2 & 0.869 & 0.755 \\
\hline Pregunta 3 & 0.788 & 0.621 \\
\hline Pregunta 4 & 0.519 & 0.269 \\
\hline Pregunta 5 & 0.768 & 0.590 \\
\hline Pregunta 6 & 0.776 & 0.602 \\
\hline Pregunta 7 & 0.784 & 0.615
\end{tabular}

Tabla 5. Índices de bondad de ajuste del análisis factorial confirmatorio para probar la idoneidad de un modelo factorial único de la versión en español de la escala de temor a la COVID-19

\begin{tabular}{|l|l|l|l|}
\hline & Modelo 1 & Modelo 2 & Modelo 3 \\
\hline Chi cuadrada & 40.489 & 30.897 & 22.802 \\
\hline$p$ & 0.000 & 0.003 & 0.029 \\
\hline CMIN -DF & 2.892 & 2.377 & 1.900 \\
\hline GFI & 0.951 & 0.962 & 0.972 \\
\hline TLI & 0.637 & 0.736 & 0.827 \\
\hline CFI & 0.768 & 0.836 & 0.901 \\
\hline RMSEA & 0.090 & 0.077 & 0.062 \\
\hline
\end{tabular}

CMIN-DF: razón de chi-cuadrada/grados de libertad; GFI: índice de bondad de ajuste; TLI: índice Tucker-Lewis; CFI: índice de ajuste comparativo; RMSEA: error de aproximación cuadrático medio.

con razón de chi-cuadrada/grados de libertad (CMIN/ $D F)=1.900(p<0.001), G F I=0.972, C F I=0.901$, RMSEA $=0.062$ (IC 95\%: 0.019-0.100) y TLI $=0.827$ (Tabla 5).

Posteriormente se calculó la fiabilidad, demostrando consistencia interna adecuada en nuestra población. Teniendo en cuenta las siete preguntas de la escala, encontramos $\alpha$ de Cronbach $=0.870$. La validez de concurrencia se realizó contrastándola con la HADS como en la versión original. Para medir la validez externa de la versión en español de la FCV-19S se utilizó el coeficiente de correlación de Pearson como medida concurrente empleando la HADS, mostrando una correlación positiva y estadísticamente significativa (rho de Pearson $=0.508, p<0.001)$ utilizando las siete preguntas (Tabla 6).

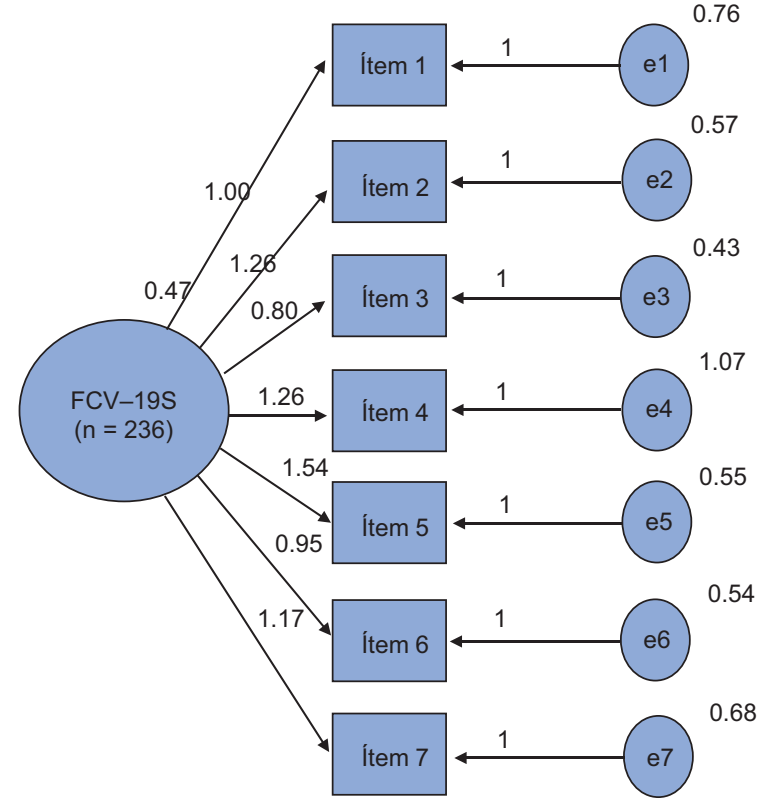

Figura 2. Modelo 1 basal sin correlación de covarianzas. FCV-19S: Escala de temor a la COVID-19.

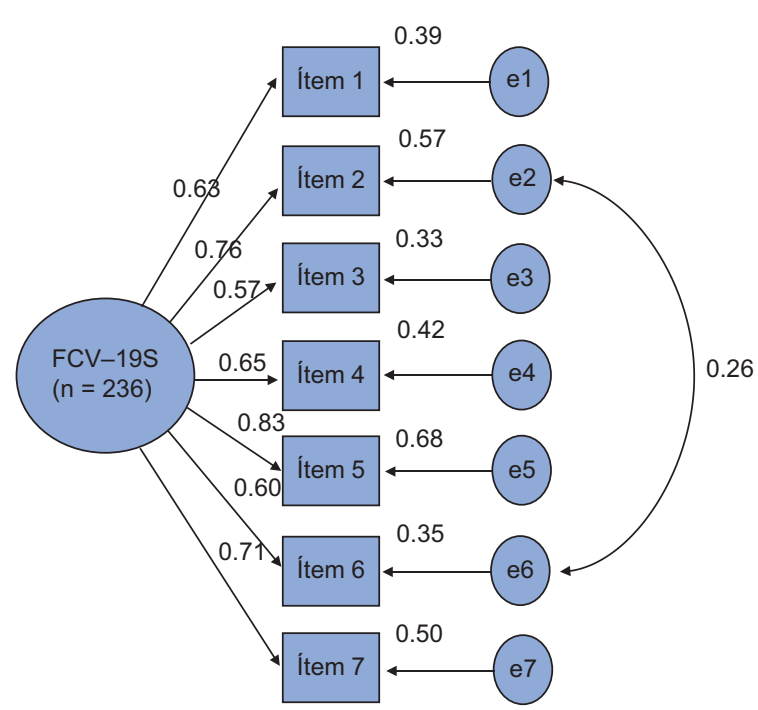

Figura 3. Modelo 2 con la correlación de covarianza entre las preguntas 3 y 6 .

\section{Discusión}

Este estudio analizó las propiedades psicométricas de la versión en español de la FCV-19S en la población general mexicana. Considerando la representatividad de la muestra, en los datos recientes del INEGI sobre las características sociodemográficas de la 


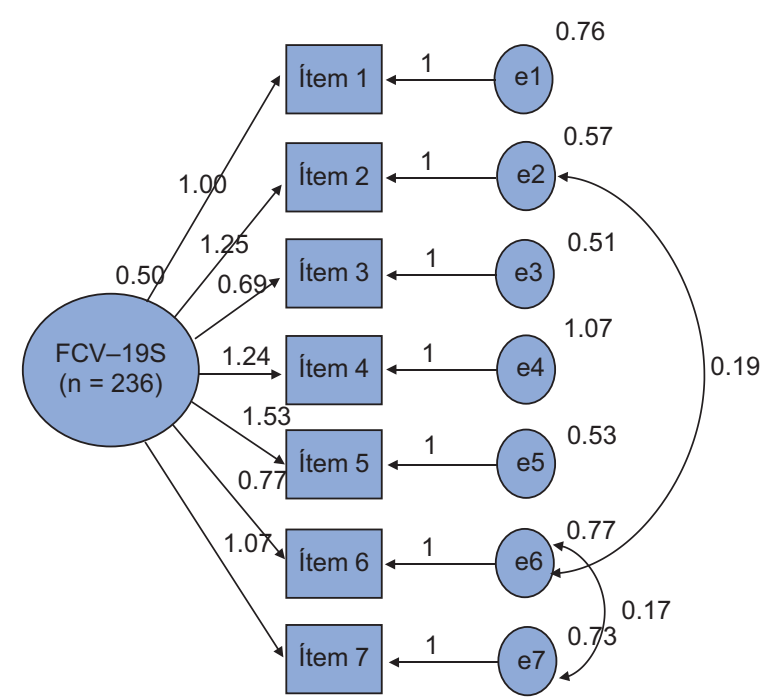

Figura 4. Modelo 3 con correlación de covarianza entre las preguntas 3, 6 y 7 .

Tabla 6. Mediciones de concurrencia

\begin{tabular}{|c|c|c|c|c|c|}
\hline & Test & Retest & $\begin{array}{l}\text { HADS } \\
\text { total }\end{array}$ \\
\hline \multirow[t]{3}{*}{ Pearson } & Test & $\begin{array}{l}\text { Coeficiente de } \\
\text { correlación } \\
\text { Sig. (2-colas) } \\
n\end{array}$ & $\begin{array}{c}1.00 \\
0.000 \\
306\end{array}$ & $\begin{array}{c}0.520^{*} \\
0.000 \\
306\end{array}$ & $\begin{array}{c}0.213^{*} \\
0.000 \\
306\end{array}$ \\
\hline & Retest & $\begin{array}{l}\text { Coeficiente de } \\
\text { correlación } \\
\text { Sig. (2-colas) } \\
n\end{array}$ & $\begin{array}{c}0.508^{*} \\
0.000 \\
306\end{array}$ & $\begin{array}{c}1.00 \\
0.000 \\
306\end{array}$ & $\begin{array}{c}0.151^{*} \\
0.008 \\
306\end{array}$ \\
\hline & $\begin{array}{l}\text { HADS } \\
\text { Total }\end{array}$ & $\begin{array}{l}\text { Coeficiente de } \\
\text { correlación } \\
\text { Sig. (2 -colas) } \\
\text { n }\end{array}$ & $\begin{array}{l}0.213^{*} \\
0.000 \\
306\end{array}$ & $\begin{array}{c}0.151^{*} \\
0.008 \\
306\end{array}$ & $\begin{array}{c}1.00 \\
0.000 \\
306\end{array}$ \\
\hline
\end{tabular}

${ }^{*} \mathrm{p}<0.001$.

HADS: Escala de ansiedad hospitalaria y depresión.

población mexicana, observamos similitud en la media de edad, ya que el $41.1 \%$ de los participantes se encontró entre 18-29 años y el $44.4 \%$ entre $30-39$ años; el nivel educativo superior en nuestra muestra $(41.1 \%)$ contrasta con el $21.6 \%$ en la población general de nuestro país, sin embargo, es más cercano al específico en la Ciudad de México ${ }^{5}$, donde el $34.6 \%$ de los habitantes tiene estudios universitarios, lo cual pudiese reflejar las diferencias sociales y culturales en nuestro país.

En cuanto a la consistencia interna, nuestro resultado de $\alpha=0.870$ es similar a las validaciones realizadas para otros idiomas como el italiano $(\alpha=0.87)^{12}$, ruso $(\alpha=0.81)^{13}$, turco $(\alpha=0.85)^{14}$, árabe $(\alpha=0.88)^{15}$, hebreo $(\alpha=0.86)^{16}$, bengalí $(\alpha=0.87)^{17}$, malayo $(\alpha=0.89)^{18}$, rumano $(\alpha=0.88)^{19}$, portugués $(\alpha=0.88)^{20}$, japones $(\alpha=0.87)^{21}$, griego $(\alpha=0.83)^{22} \mathrm{e}$ inglés $(\alpha=0.84 \text { y } 0.86 \text { en diferentes muestras })^{23}$.

Nuestros resultados son similares a las validaciones realizadas en español en otros contextos como la población peruana $(\alpha=0.87)^{24}$ y estudiantes universitarios españoles $(\alpha=0.86)^{25}$.

La media del puntaje total de la FCV-19S en nuestra población fue de $17.6 \pm 6.6$, similar a los italianos 16.8 $\pm 6.6^{12}$ y los rusos $17 \pm 4.7^{13}$. Además, considerando el sexo, observamos diferencias significativas entre las mujeres $(19.09 \pm 6.67)$ y los hombres (14.99 \pm $5.62)$, similares a los hallazgos en otras poblaciones como en Bangladesh (mujeres $22.75 \pm 5.65$ y hombres $20.29 \pm 5.90)^{17}$ o en Rusia (mujeres $17.7 \pm 4.6 \mathrm{y}$ hombres $15.8 \pm 4.6)^{13}$. La puntuación total reportada en el FCV-19S fue más alta en las mujeres, lo cual corresponde a los informes internacionales sobre salud mental con una mayor prevalencia de los trastornos de ansiedad y depresión en las mujeres.

Con respecto a la carga factorial de los elementos de nuestra versión (rango: 0.519-0.869) fueron similares a los de otras, como la italiana $(0.68-0.90)^{12}$, árabe $(0.62-0.84)^{15}$, bengalí $(0.72-0.80)^{17} y$ peruana en el modelo de un factor $(0.595-0.766)^{24}$.

De hecho, el análisis factorial confirmatorio en nuestros resultados de $\mathrm{CFI}=0.901$ y $\mathrm{RMSEA}=0.062$ (IC 95\%: 0.019-0.100) son comparables a los de otras versiones que utilizaron un modelo unifactorial con correlación en varios elementos, como la árabe $(\mathrm{CFI}=0.995, \mathrm{RMSEA}=0.059, \mathrm{IC} 95 \%: 0.037-0.083)^{15}$, bengalí $(C F I=0.964 \text {, RMSEA }=0.071)^{17} \mathrm{e}$ italiana $(\mathrm{CFI}=0.99, \mathrm{RMSEA}=0.069, \mathrm{IC} 95 \%: 0.032-0.105)^{12}$.

En nuestra población, las puntuaciones más bajas se encontraron en las preguntas 3,6 y 7 , que evalúan síntomas somáticos como sudoración palmar, insomnio y palpitaciones, lo cual es similar a los hallazgos en otras poblaciones como la italiana ${ }^{12}$, turca $^{14} 0$ bengalíi ${ }^{\prime 7}$.

Por lo anterior es importante realizar una validación y adaptación cultural en cada población, teniendo en cuenta las diferencias de sexo y edad, para reflejar la realidad de cada contexto sociocultural, permitiendo una medición fiable sobre el temor relacionado con la COVID-19.

La validación del FCV-19S en su versión en español para su aplicación en la población mexicana demuestra que es un instrumento corto, fácil de aplicar e interpretar, que permite un tamizaje identificando a 
individuos en riesgo de desarrollar trastornos de ansiedad y depresión, con el fin de iniciar el tratamiento oportuno.

\section{Fortalezas y limitaciones}

Los participantes mostraron resistencia inicial a participar en el estudio, lo cual asociamos al contexto sociocultural en nuestro país, donde la atención de salud mental no es una prioridad.

Como limitación reconocemos que la muestra general puede considerarse representativa, aunque heterogénea dado la edad, el sexo, el nivel académico y el estado civil; somos conscientes de que no se siguió ningún enfoque formal para garantizarlo.

Por otro lado, este análisis no incluyó el diagnóstico previo de trastornos mentales como depresión, trastornos de ansiedad o de la personalidad que en estudios posteriores deben tenerse en cuenta al reconocer a subgrupos con mayor vulnerabilidad ante situaciones estresantes. Al mismo tiempo, se trata de un estudio transversal, por lo que un seguimiento longitudinal posterior podría ser bastante enriquecedor. A pesar de estas limitaciones, este estudio muestra información útil sobre el uso de la FCV-19S en su versión en español para su aplicación en la población general de México. Además, consideramos indispensable realizar un análisis posterior en los trabajadores del área de la salud que participan en los equipos de respuesta COVID, pues se encuentran estrechamente en contacto con los enfermos, con el fin de validar su uso específico en este grupo de población.

\section{Conclusión}

La versión en español del FCV-19S mostró una fiabilidad y consistencia interna adecuadas, además de una buena correlación en la puntuación de las mismas mediciones y correlación posteriores con el HADS, confirmando la validez de convergencia en nuestro análisis. Al mismo tiempo, los resultados obtenidos son similares a los reportados por otros estudios en poblaciones de habla hispana, lo que sugiere que es una escala adecuada con propiedades psicométricas adecuadas para la detección inicial en la población general en México.

\section{Agradecimientos}

Los autores agradecen a Susan Drier-Jonas por su apoyo con el manuscrito.

\section{Financiamiento}

Esta investigación no ha recibido ninguna beca específica de agencias de los sectores públicos, comercial o sin ánimo de lucro.

\section{Conflicto de intereses}

Los autores no tienen conflicto de intereses para declarar.

\section{Responsabilidades éticas}

Protección de personas y animales. Los autores declaran que para esta investigación no se han realizado experimentos en seres humanos ni en animales.

Confidencialidad de los datos. Los autores declaran que han seguido los protocolos de su centro de trabajo sobre la publicación de datos de pacientes.

Derecho a la privacidad y consentimiento informado. Los autores han obtenido el consentimiento informado de los pacientes y/o sujetos referidos en el artículo. Este documento obra en poder del autor de correspondencia.

\section{Bibliografía}

1. World Health Organization. WHO Director-General's opening remarks at the media briefing on COVID-19 - 11 March 2020 [Internet]. World Health Organization; 2020 [consultado: 15 de marzo de 2020]. Disponible en: https://www.who.int/dg/speeches/detail/who-director-general-s-openingremarks-at-the-media-briefing-on-covid-19---11-march-2020

2. Dirección General de Epidemiología, Secretaría de Salud de México. Covid-19 México. Información General [Internet]. México: Dirección General de Epidemiología, Secretaría de Salud; 29 de enero de 2021 [consultado: 18 de marzo de 2021]. Disponible en: https://datos.covid-19.conacyt.mx

3. Instituto Nacional de Estadística y Geografía. Características de las defunciones registradas en México durante enero a agosto de 2020 [Internet]. México: Instituto Nacional de Estadística y Geografía (INEGI), Comunicado de prensa Núm. 61/21; 27 de enero de 2021 [consultado: 18 de marzo de 2021]. Disponible en: https://www.inegi.org.mx/contenidos/saladeprensa/boletines/2021/EstSociodemo/DefuncionesRegistradas2020_Pnles.pdf

4. Dirección General de Epidemiología, Secretaría de Salud. Informe semanal para la vigilancia epidemiológica de muertes maternas (4 de enero de 2021-semana epidemiológica 53) [Internet]. México: Dirección General de Epidemiología, Secretaría de Salud [consultado: 18 de marzo de 2021]. Disponible en: https://www.gob.mx/salud/documentos/informes-semanales-para-la-vigilancia-epidemiologica-de-muertes-maternas-2020. Accessed: March 18, 2021.

5. Instituto Nacional de Estadística y Geografía. Panorama sociodemográfico de México. Censo de Población y Vivienda 2020 [Internet]. México: Instituto Nacional de Estadística y Geografía (INEGI); enero 2021 [consultado: 18 de marzo de 2021]. Disponible en: https://www.inegi.org.mx

6. Li YC, Bai WZ, Hashikawa T. The neuroinvasive potential of SARS-CoV2 may play a role in the respiratory failure of COVID-19 patients. J Med Virol. 2020;92(6):552-5.

7. Moreno C, Wykes T, Galderisi S, Nordentoft M, Crossley N, Jones N How mental health care should change as a consequence of the $\mathrm{CO}$ VID-19 pandemic. Lancet Psychiatry. 2020;7(9):813-24.

8. Ahorsu DK, Lin CY, Imani V, Saffari M, Griffiths MD, Pakpour AH. The fear of COVID-19 scale: Development and initial validation. Int J Ment Health Addict. 2020 Mar 27:1-9. doi: 10.1007/s11469-020-00270-8. Online ahead of print. 
9. Soraci P, Ferrari A, Abbiati FA, del Fante E, de Pace R, Urso A, et al. Validation and psychometric evaluation of the Italian Version of the Fear of COVID-19 Scale. Int J Ment Health Addict. 2020 May 4;1-10. doi: 10.1007/s11469-020-00277-1. Online ahead of print.

10. Reznik A, Gritsenko V, Konstantinov V, Khamenka N, Isralowitz R. COVID19-fear in Eastern Europe: Validation of the Fear of COVID-19 Scale. Int J Ment Health Addict. 2020 May 12:1-6. doi: 10.1007/s11469-02000283-3. Online ahead of print.

11. Haktanir A, Seki T, Dilmac B. Adaptation and evaluation of Turkish Fear of COVID-19 Scale. Death Stud. 2020;29:1-9.

12. Alyami M, Henning M, Krägeloh $C$, Alyami H. Psychometric evaluation of the Arabic version of the Fear of COVID-19 Scale. Int J Ment Health Addict. 2020 May 16:1-14. doi: 10.1007/s11469-020-00316-x. Online ahead of print.

13. Tzur BD, Grossman GA, Bloch Y, Mayer Y, Shiffman N, Mendlovi S. Fear of COVID-19 scale. Psychometric characteristics, reliability and validity in Israeli population. Psychiatry Res. 2020;289:113100.

14. Sakib N, Bhuiyan AKMI, Hossain S, AI Mamun F, Hosen I, Hasnat Abdullah A, et al. Psychometric validation of the Bengla Fear of COVID-19 Scale Confirmatory Factor Analysis and Rasch Analysis. Int J Ment Health Addict. 2020 May 11:1-12. doi: 10.1007/s11469-020-00289-x. Online ahead of print.

15. Pang NTP, Kamu A, Hambali NLB, Mun HC, Kassim MA, Mohamed NH et al. Malay version of the Fear of COVID-19 Scale: Validity and reliability. Int J Ment Health Addict. 2020 Jul 3:1-10. doi: 10.1007/s11469-02000355-4. Online ahead of print.

16. Stănculescu E. Fear of COVID-19 in Romania: Validation of the Romanian version of the Fear of COVID-19 Scale using graded response model analysis. Int J Ment Health Addict. 2021 Jan 6;1-16. doi: 10.1007/ s11469-020-00428-4. Online ahead of print.

17. Magano J, Vidal DG, Sousa HFPE, Dinis MAP, Leite Â. Validation and psychometric properties of the Portuguese version of the Coronavirus Anxiety Scale (CAS) and Fear of COVID-19 Scale (FCV-19S) and associations with travel, tourism and hospitality. Int J Environ Res Public Health. 2021;18(2):E427.
18. Wakashima K, Asai K, Kobayashi D, Koiwa K, Kamoshida S, Sakuraba M. The Japanese version of the Fear of COVID-19 Scale: Reliability, validity, and relation to coping behavior. PLoS One. 2020;15(11):e0241958.

19. Nikopoulou VA, Holeva V, Parlapani, E, Karamouzi P, Voitsidis P, Porfyri GN, et al. Mental health screening for COVID-19: a proposed cutoff score for the Greek version of the Fear of COVID-19 Scale (FCV-19S). 2020 Nov 10;114. doi: 10.1007/s11469-020-00414-w. Online ahead of print.

20. Winter T, Riordan BC, Pakpour AH, Griffiths MD, Mason A, Poulgrain JW, et al. Evaluation of the English Version of the Fear of COVID-19 Scale and Its relationship with behavior change and political beliefs. Int J Ment Health Addict. 2020 Jun 15;1-11. doi: 10.1007/s11469-020-00342-9. Online ahead of print.

21. Huarcaya-Victoria J, Villareal-Zegarra D, Podesta A, Luna-Cuadros MA. Psychometric Properties of a Spanish Version of the Fear of COVID-19 Scale in General Population of Lima, Peru. Int J Ment Health Addict. 2020 Jun 22:1-14. doi: 10.1007/s11469-020-00354-5. Online ahead of print.

22. Martínez- Lorca M, Martínez-Lorca A, Criado-Álvarez JJ, Cabañas Armesilla MD, Latorre JM. The fear of COVID-19 scale: Validation in Spanish university students. Psychiatry Research. 2020;293:113350.

23. García-Reyna, B, Castillo-García,GB, Barbosa-Camacho, FJ, Cervantes-Cardona GA, Cervantes-Pérez E, Torres-Mendoza BM, et al. Fear of COVID-19 Scale for Hospital Staff in Regional Hospitals in México: a brief report. Int J Ment Health Addict. 2020 Nov 4:1-12. doi: 10.1007/ s11469-020-00413-x. Online ahead of print.

24. Zigmond AS, Snaith RP. The Hospital Anxiety and Depression Scale. Acta Psychiatrica Scandinavica. 1983;67(6):361-70.

25. Villegas Pérez GC. Reporte de experiencia profesional. Tesis de maestría en Psicología. México: Facultad de Psicología, Universidad Nacional Autónoma de México; 2004.

26. Vázquez GO, Benjet C, Júarez GF, Rojas CE, Riveros RA, Aguilar PJL, et al. Propiedades psicométricas de la Escala Hospitalaria de Ansiedad y Depresión en una población de pacientes oncológicos mexicanos. Salud Ment. 2015;38(4):253-8. 